\title{
Magnetic Resonance Imaging in Peripartum Encephalopathy: A Pictorial Review
}

\author{
Duraipandi Manjubashini ${ }^{1}$ Krishnan Nagarajan \\ Vaibhav Wadwekar ${ }^{3}$ Sunil K. Narayan ${ }^{3}$ \\ 1Department of Radiodiagnosis, Jawaharlal Institute of \\ Postgraduate Medical Education \& Research (JIPMER), \\ Puducherry, India \\ 2Department of Obstetrics and Gynecology, Jawaharlal Institute of \\ Postgraduate Medical Education \& Research (JIPMER), \\ Puducherry, India \\ ${ }^{3}$ Department of Neurology, Jawaharlal Institute of Postgraduate \\ Medical Education \& Research (JIPMER), Puducherry, India
}

\author{
Mohan Amuthabarathi ${ }^{1}$ Dasari Papa
}

J Neurosci Rural Pract 2021;12:402-409.

\begin{abstract}
Address for correspondence Krishnan Nagarajan, MD, DM, Department of Radiodiagnosis, Jawaharlal Institute of Postgraduate Medical Education \& Research (JIPMER), Puducherry 605006, India (e-mail: Iknagarajan1@gmail.com).
\end{abstract}

\begin{abstract}
Keywords

- peripartum encephalopathy

- magnetic resonance imaging

- posterior reversible encephalopathy

- postpartum angiopathy

Acute neurological problems are significant disorders of pre- and postpartum period in women. We analyzed the magnetic resonance imaging (MRI) profile of patients presenting with peripartum encephalopathy over 2 years. Of 51 patients, MRI was abnormal in 40 patients and normal in 11 patients. Posterior reversible encephalopathy (13/40), cerebral venous thrombosis (6/40), and postpartum angiopathy (3/40) are the three most common causes of peripartum encephalopathy as identified in MRI. The other less common but important etiology include HELLP (Hemolysis, Elevated Liver enzymes and Low Platelet) syndrome (2), osmotic demyelination (2), antiphospholipid syndrome (2), tubercular meningitis/cerebritis (3), pituitary hyperplasia with hemorrhage (2), postictal edema (2), cerebellitis (1), transient splenial lesion (1), and changes of old trauma and stroke (one each).
\end{abstract}

\section{Introduction}

Acute neurological diseases occurring in peripartum ( 2 weeks before and 6 weeks after labor) period are serious complications that can lead to maternal morbidity and mortality. There are a variety of causes for peripartum encephalopathy, which may be unique to pregnant/postpartum state such as preeclampsia, or labor-associated neurological or other nonobstetric causes. The clinical presentations of peripartum encephalopathy include seizure, headache, altered sensorium, or acute psychosis. Acute neurological diseases in pregnancy are associated with many obstetric and neonatal complications. Neuroimaging in these patients has a vital role in identifying the abnormality and in the management. ${ }^{1-6}$ Magnetic resonance imaging (MRI) is the modality

DOI https://doi.org/ 10.1055/s-0041-1727300 ISSN 0976-3147 of choice in the pregnancy period as it is a nonradiation investigative modality. Early diagnosis and management are required in these cases and the management.

\section{Methods}

We reviewed the clinical and MR neuroimaging features of peripartum women presenting with encephalopathy with or without neurological deficits, seizures, etc. from January 2016 to December 2017. A total of 52 women aged 18 years and above admitted with encephalopathy to the labor room or obstetric ward in the hospital were included in the study. They presented with features of encephalopathy-altered sensorium-along with other features including seizures, headache, or focal deficits. They underwent MRI within 72 hours 
of initial presentation, as a part of their routine investigation. MRI was done in 1.5 Tesla equipment (Avanto Magnetom Siemens Erlangen, Germany) using routine sequencesfluid-attenuated inversion recovery (FLAIR) axial, T2-weighted coronal, T1-weighted sagittal sequences, diffusion-weighted imaging, MR angiography of circle of Willis, and gradient-echo T2*-weighted sequence to rule out hemorrhage.

\section{Results}

A total of 51 peripartum encephalopathy cases, comprising 40 cases with abnormal MRI findings and 11 with normal MRI findings, were studied (-Table 1). Out of 40 with abnormal MRI, 13 cases were with posterior reversible encephalopathy syndrome (PRES), 6 with cerebral venous thrombosis (CVT), 3 with postpartum angiopathy, 2 with hemolysis, elevated liver enzymes, and low platelets (HELLP) syndrome, 3 with infection/tubercular meningitis, 2 with antiphospholipid syndrome/vasculitis, 2 with osmotic demyelination syndrome, 2 with pituitary lesions (one each hemorrhage into pituitary and macroadenoma with hemorrhage), 2 with postictal edema, 1 with transient splenial lesion, 1 with spontaneous intracranial hypotension, 1 with old stroke, and 1 with gliosis of old trauma.

\section{Discussion}

\section{Posterior Reversible Encephalopathy Syndrome}

Hypertension and related disorders of pre-eclampsia and eclampsia are the commonest causes of medical complications in pregnancy, with a varying incidence of 6 to $8 \% .{ }^{7,8}$ Since the involvement was more in the posterior parieto-occipital regions and to a large extent they are reversible, this clinicoradiological entity is called PRES. Near-complete resolution of clinical features and radiologic findings is usually seen after treatment with antihypertensive drugs and, if prepartum, with delivery. In our group, PRES-related changes were noticed in 13 patients (31.7\%). Abnormal findings in MRI were vasogenic edema involving the parieto-occipital lobes in 11 cases (84.6\%), temporal lobes in 2 cases (15.4\%), cerebellum in 3 cases (23.1\%), brainstem in 2 cases (15.4\%), basal ganglia in 4 cases (30.8\%), and frontal lobes in 8 cases (61.5\%). The parietal lobe is involved in all the 13 cases (100\%). Typically, the lesions involve the white matter, but rarely gray matter can also be involved (-Fig. 1). Atypical findings in PRES include central location of lesions sparing the posterior fossa, presence of infarcts and lesions associated with hemorrhage. Diffusion-weighted MR may show occasional lesions with cytotoxic edema as restricted diffusion and may lead to areas of gliosis.

\section{Cerebral Venous Thrombosis}

Pregnancy and the postpartum period are relatively hypercoagulable states with increased risk of CVT.,9-11 The risk factors for development of CVT include relative immobility, fluid-electrolyte changes, infection, and inflammatory disorders. Pregnancy is state of physiological hypercoagulability and can lead to CVT in a few women. Obesity, hypertension, cesarean section, hyperemesis, dehydration, oral contraceptive pills, and prolonged bed rest are other predisposing factors. The clinical presentation of CVT depends upon the

Table 1 Magnetic resonance imaging (MRI) diagnosis in peripartum encephalopathy

\begin{tabular}{|l|l|l|}
\hline & MRI Diagnosis & $\begin{array}{l}\text { No. of } \\
\text { patients } \\
\text { (percentage) }\end{array}$ \\
\hline 1 & $\begin{array}{l}\text { Posterior reversible encephalopathy } \\
\text { syndrome (PRES) }\end{array}$ & $13(31.7)$ \\
\hline 2 & Cerebral venous thrombosis (CVT) & $6(14.6)$ \\
\hline 3 & $\begin{array}{l}\text { Postpartum angiopathy (PPA)/reversi- } \\
\text { ble cerebral vasoconstriction syndrome } \\
\text { (RCVS) }\end{array}$ & $3(7.3)$ \\
\hline 4 & $\begin{array}{l}\text { Hemolysis, elevated liver enzymes, and } \\
\text { low platelet syndrome }\end{array}$ & $2(4.9)$ \\
\hline 5 & Osmotic demyelination & $2(4.9)$ \\
\hline 6 & $\begin{array}{l}\text { Antiphospholipid syndrome (APLAS)/ } \\
\text { vasculitis }\end{array}$ & $2(4.9)$ \\
\hline 7 & Infection/tubercular meningitis & $3(7.3)$ \\
\hline 8 & Pituitary lesions & $2(4.9)$ \\
\hline 9 & Postictal edema & $2(4.9)$ \\
\hline 10 & Transient splenial lesions (TSL) & $1(2.4)$ \\
\hline 11 & Cerebellitis & $1(2.4)$ \\
\hline 12 & Others (old stroke, trauma) & $3(7.3)$ \\
\hline & Normal MRI & 11 \\
\hline & & 40 \\
\hline
\end{tabular}
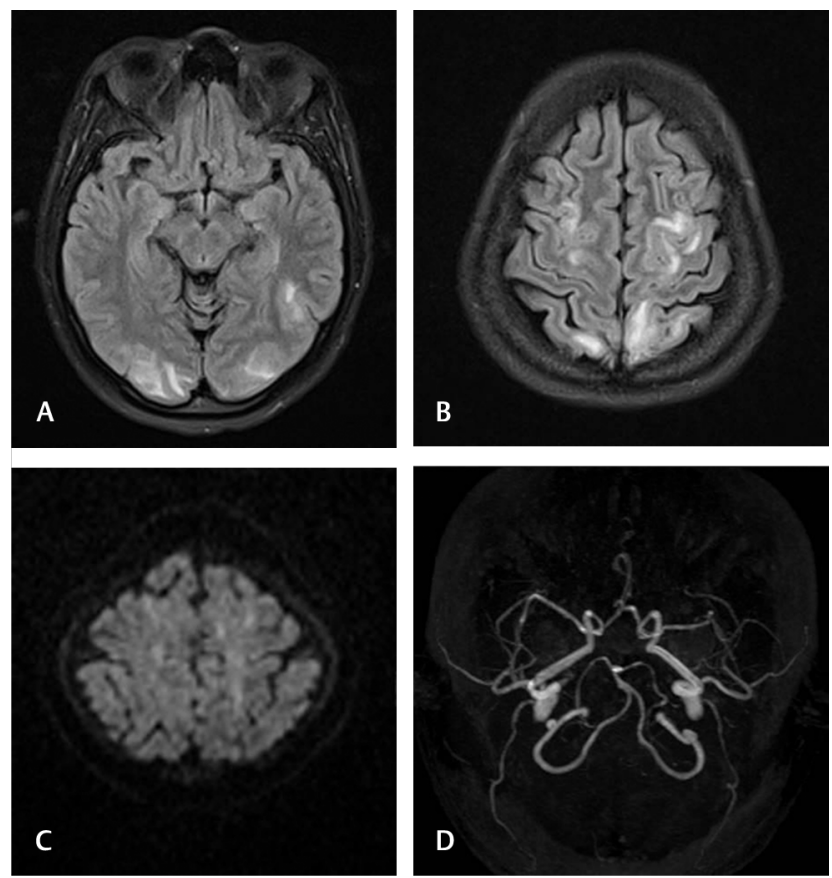

Fig. 1 Posterior reversible encephalopathy syndrome. A 21-year-old primigravida presented with postpartum encephalopathy. Fluid-attenuated inversion recovery axial sections ( $\mathbf{A}$ and $\mathbf{B}$ ) showing occipital and frontoparietal subcortical white matter hyperintensities. No diffusion restriction is noted (C). Magnetic resonance angiogram is normal (D). 

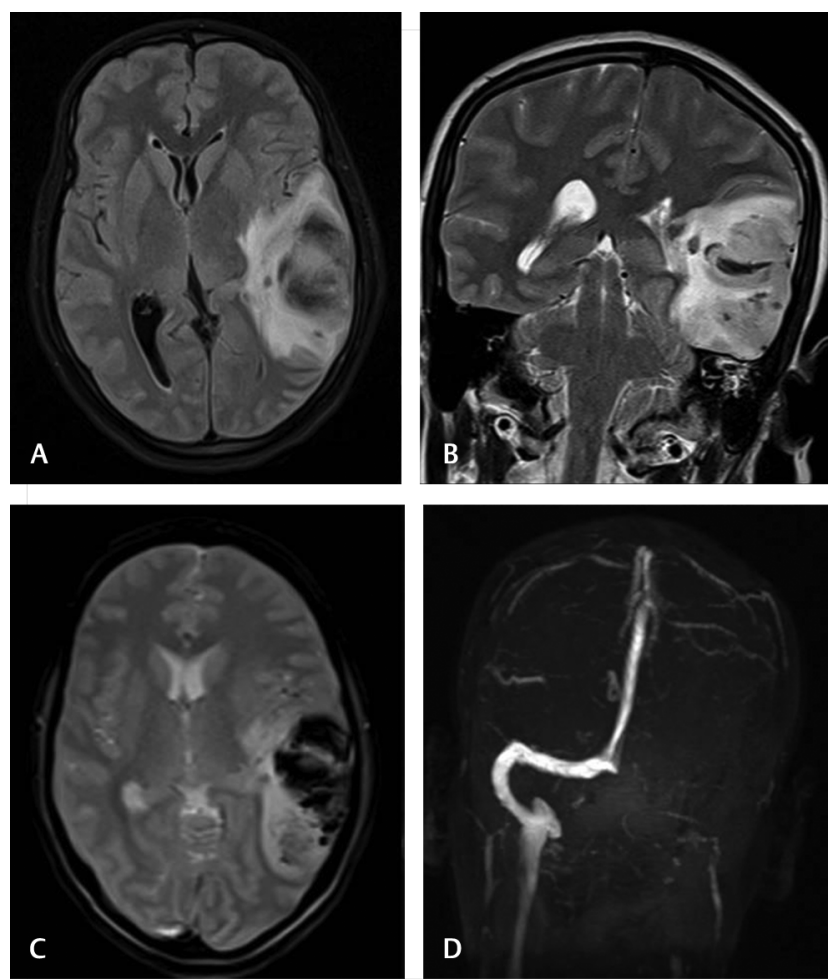

Fig. 2 Cerebral venous thrombosis. A 27-year-old female with second pregnancy presented before labor with altered sensorium. Fluid-attenuated inversion recovery axial (A), T2 coronal sections showing left temporoparietal hemorrhagic infarction due to venous thrombosis (B). Gradient-echo T2* (C) images showing hypointense hemorrhage with absent left transverse sinus in magnetic resonance venogram (D).

territory of the affected vessel, the usual presentation being headache. It can also present with seizures and papilledema, secondary to increased intracranial pressures. MRI with MR venography delineates the intracranial venous sinuses. The superior sagittal sinus and transverse sinuses are most commonly involved. When the gray matter is involved, the patients may present with either seizures or even focal neurological deficits such as hemiparesis or aphasia. In our study, cerebral venous thrombosis was found in six cases (14.6\%). Transverse sinus was involved in three cases (50\%), sigmoid sinus was thrombosed in three cases (50\%), and two cases (33\%) had cortical venous thrombosis. The venous infarct was associated with hemorrhage in four cases $(67 \%)$ ( -Fig. 2).

\section{Postpartum Angiopathy}

Postpartum Angiopathy is a subset of reversible cerebral vasoconstriction syndrome (RCVS). RCVS is a recently described entity with features of reversible narrowing of multiple cerebral arteries. ${ }^{12,13}$ It may be preceded by severe headaches with or without neurological deficit. It can also present with subarachnoid hemorrhage (associated with "thunderclap" headache), seizures, or migrainous headache. Occasionally, it may result in ischemic or hemorrhagic stroke with or without permanent sequelae. Imaging findings of RCVS include multifocal segmental narrowing of intracranial arteries, which usually resolves in 12 weeks. The parenchymal involvement is usually located in areas of anterior circulation. Sometimes presence of vasospasm is the only imaging finding. In this study, three (7.3\%) were found to have RCVS. Among them, one each had vasogenic edema, infarct, and hemorrhagic infarct ( $\boldsymbol{- \text { Fig. }} \mathbf{3}$ ). Vessel narrowing was noted in only one patient. In fact, MRI may be initially even be normal or may have variable presentation even mimicking PRES. ${ }^{14-16}$ Fugate et $\mathrm{al}^{15}$ reviewed retrospectively patients showing vasoconstriction in the postpartum period and found among 18 patients, 5 had normal MRI, 7 had hemorrhage, and 6 each had infarcts and vasogenic edema.

\section{HELLP Syndrome}

HELLP syndrome is diagnosed based on features of Hemolysis, Elevated Liver enzymes, and Low Platelets syndrome. ${ }^{17,18}$ MRI findings may overlap with those of PRES, but lesions frequently involve brainstem, deep gray structures of basal ganglia and thalami, apart from the cerebral hemispheres. ${ }^{19}$ Thrombotic microangiopathy in HELLP syndrome can cause lacunar infarcts/lesions in brain stem, basal ganglia, and thalami. We had two patients with features of HELLP syndrome-in which both had hemolytic anemia but one had elevated liver enzymes and other one had low platelet counts. MRI showed FLAIR hyperintensities with mild diffusion restriction involving the brainstem, thalami, and internal capsules ( - Fig. 4). The lesions resolved on follow-up in one patient and another patient succumbed.

\section{Osmotic Demyelination Syndrome}

Osmotic demyelination syndrome (ODS) is thought to result from "rapid" correction of hyponatremia and is caused by demyelination in the pontine tracts/neurons, although extrapontine sites like the internal capsule, basal ganglia, and cerebellum may also be involved. Hyponatremia, the inciting electrolyte abnormality, can occur in pregnancy. ${ }^{20,21}$ The classical symptoms include neurological deficit and pseudobulbar palsy, secondary to damage involving the corticospinal and corticobulbar paths. We had two cases of OSD: one case had pontine involvement and the other case had pontine and extrapontine involvement [ - Fig. 5].

\section{Antiphospholipid Syndrome/Vasculitis}

Pregnancy and puerperium are considered high-risk for those with antiphospholipid syndrome and may present with arterial or venous thrombosis. ${ }^{22,23}$ We had two patients with complete internal carotid artery occlusions ( - Fig. 6)-one 

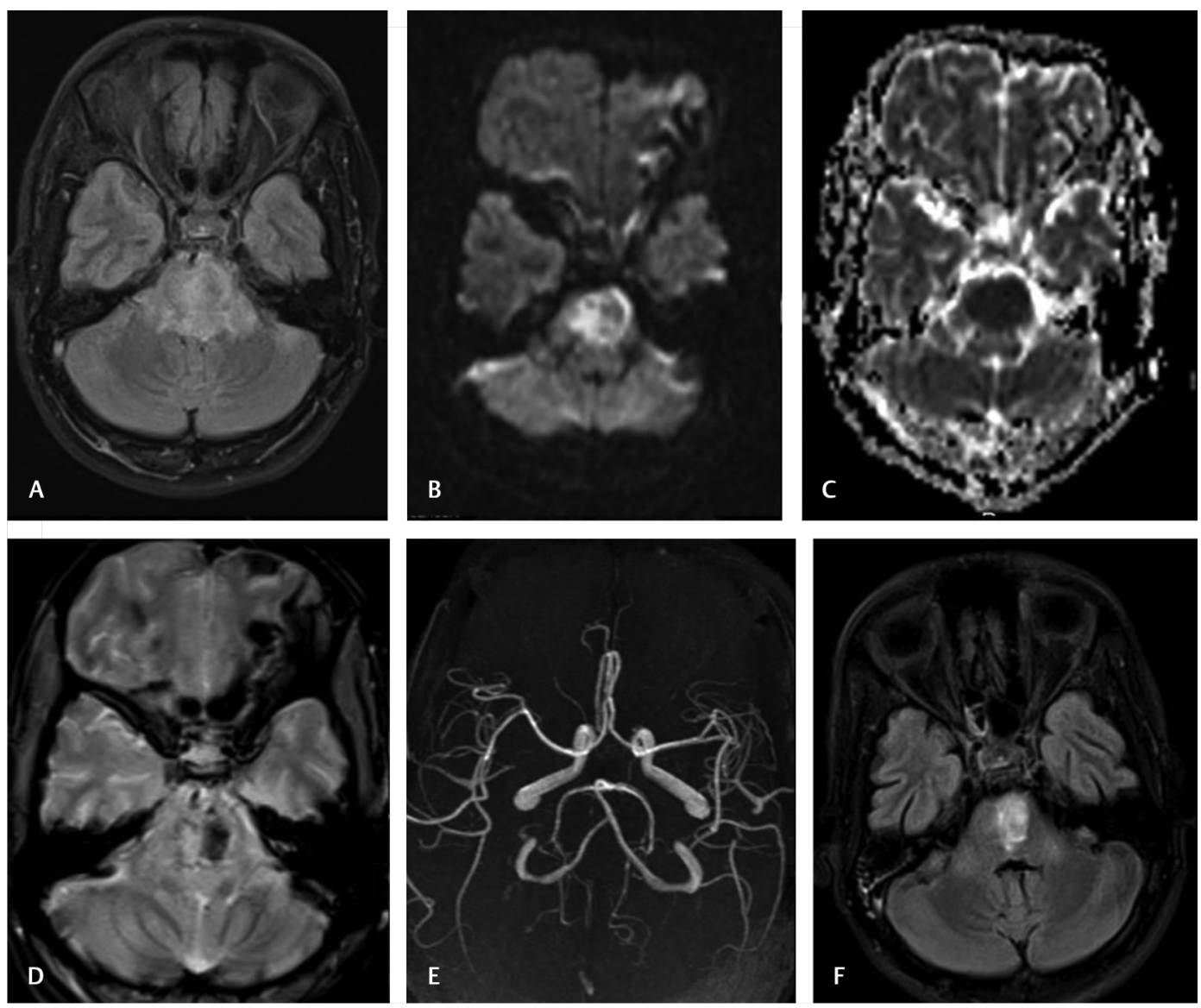

Fig. 3 Postpartum angiopathy. A 23-year-old primigravida presented with encephalopathy. Fluid-attenuated inversion recovery axial, diffusion $(\mathbf{A}, \mathbf{B})$ and apparent diffusion coefficient (C) images shows acute infarction in the pons with areas if hemorrhage in gradient-echo T2* image (D). Magnetic resonance angiogram (E) is normal. Follow-up magnetic resonance imaging (F) after 6 weeks shows partial resolution.
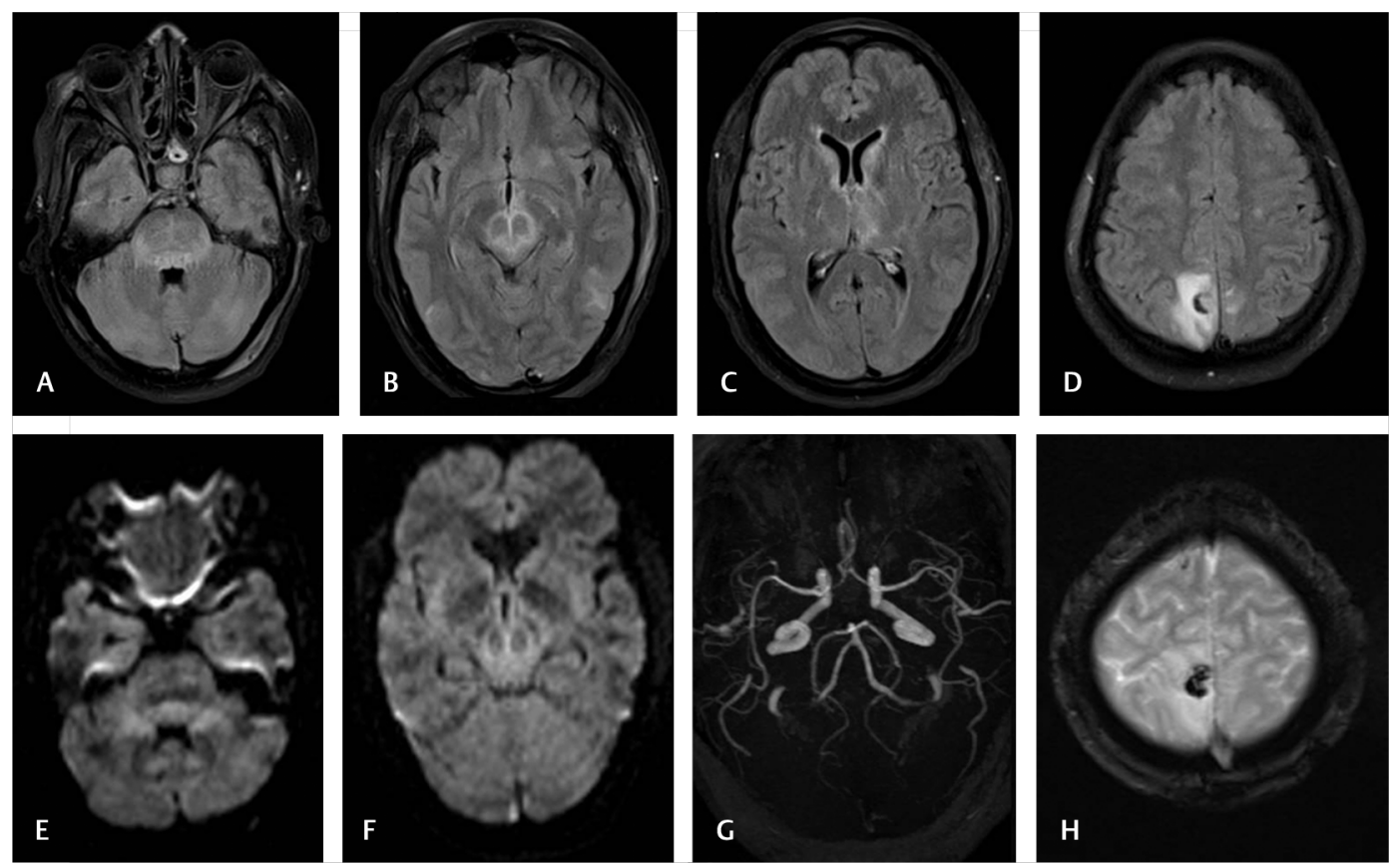

Fig. 4 Hemolysis, elevated liver enzymes, and low platelet. A 25-year-old primigravida presented 2 days before labor with encephalopathy, elevated liver enzymes, and mild reduction in platelet count. Fluid-attenuated inversion recovery axial sections (A-D) showing hyperintensities in pons, midbrain, left caudate, thalamus, and right parietal regions. Subtle diffusion restriction noted in pons and midbrain lesions ( $\mathbf{E}$ and $\mathbf{F}$ ). Magnetic resonance angiogram $(\mathbf{G})$ is normal. Gradient-echo T28 image $(\mathbf{H})$ shows hemorrhagic component in right parietal lesion. 

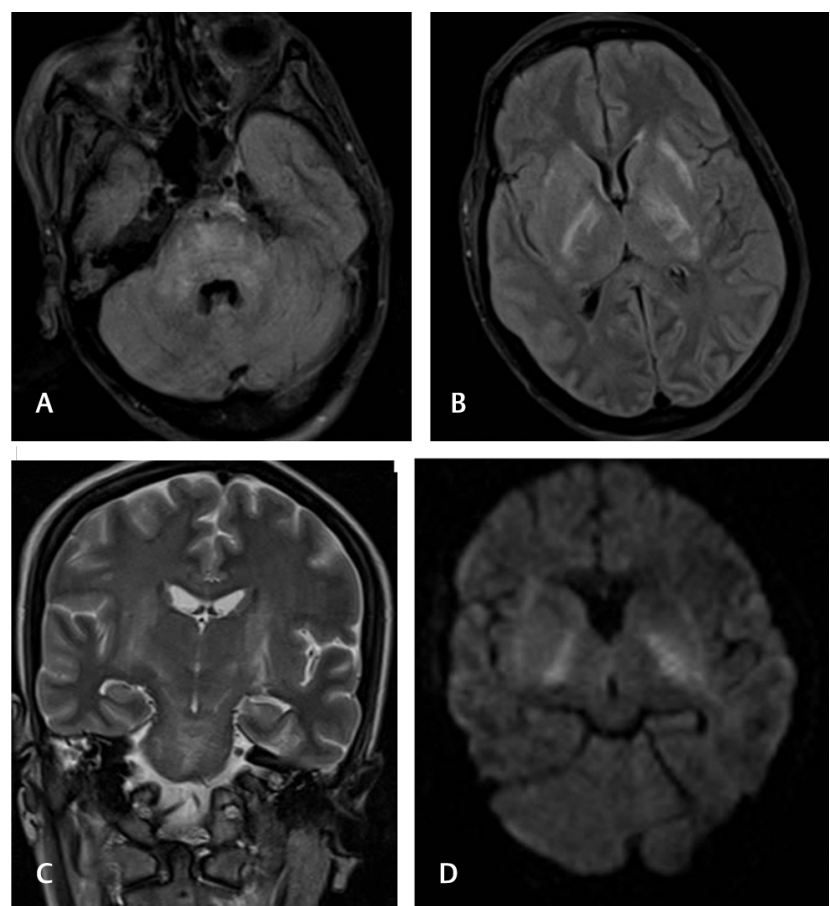

Fig. 5 Osmotic demyelination syndrome. A 28-year-old G2P1 presented 2 weeks after delivery with encephalopathy and low sodium. Fluid-attenuated inversion recovery axial (A and B) and T2 coronal images showing hyperintensities in pontine and extrapontine locations suggestive of osmotic demyelination (C). Mild diffusion restriction noted in bilateral internal capsule regions (D).
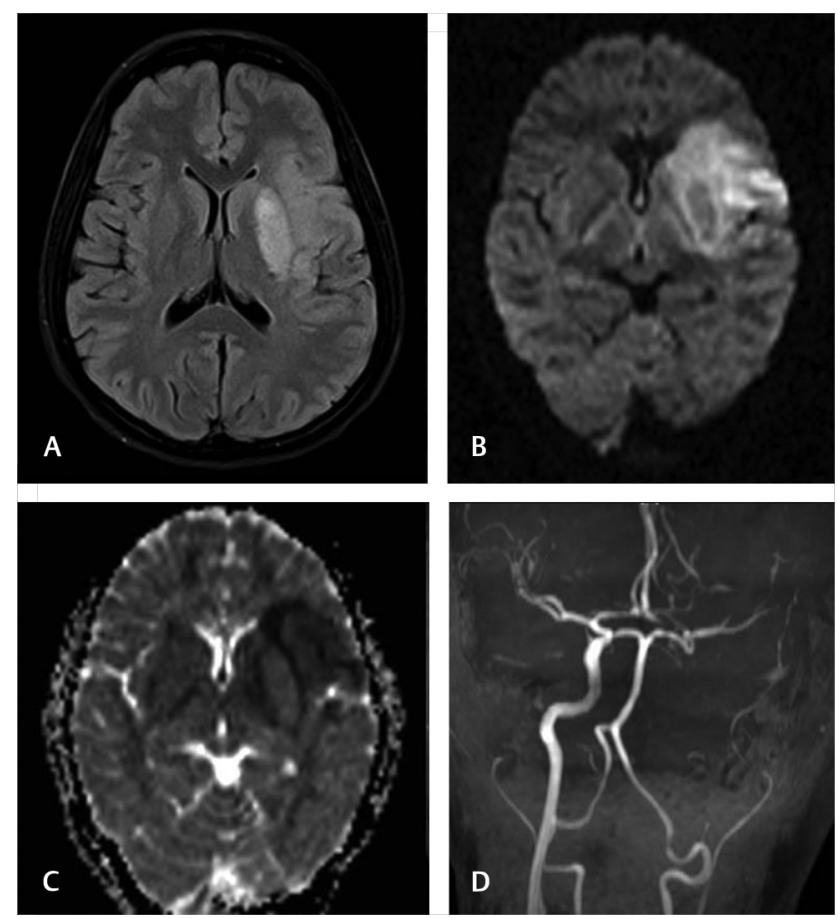

Fig. 6 Antiphospholipid syndrome. A28-year-old G1P1 presented with right hemiplegia and underwent caesarean section. Fluid-attenuated inversion recovery axial, diffusion, and apparent diffusion coefficient images show acute infarct in left basal ganglia, insula, and adjacent frontotemporal region. Magnetic resonance angiogram shows left internal carotid artery occlusion.
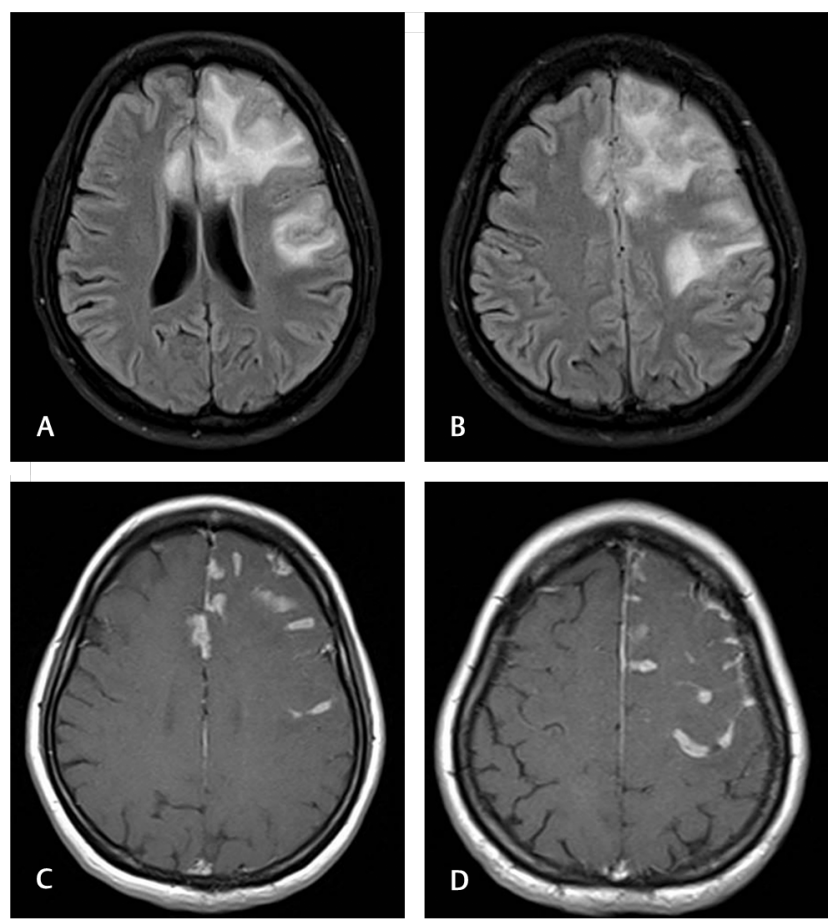

Fig. 7 Tubercular cerebritis. A 22-year-old G3P1L1A1 presented 4 days before labor with seizures and altered sensorium. Magnetic resonance imaging fluid-attenuated inversion recovery axial ( $\mathbf{A}$ and B) and post-contrast T1 (C and D) images showing enhancing focal cerebritis, meningitis, and few granulomata. Follow-up after antitubercular treatment, lesions completely resolved.
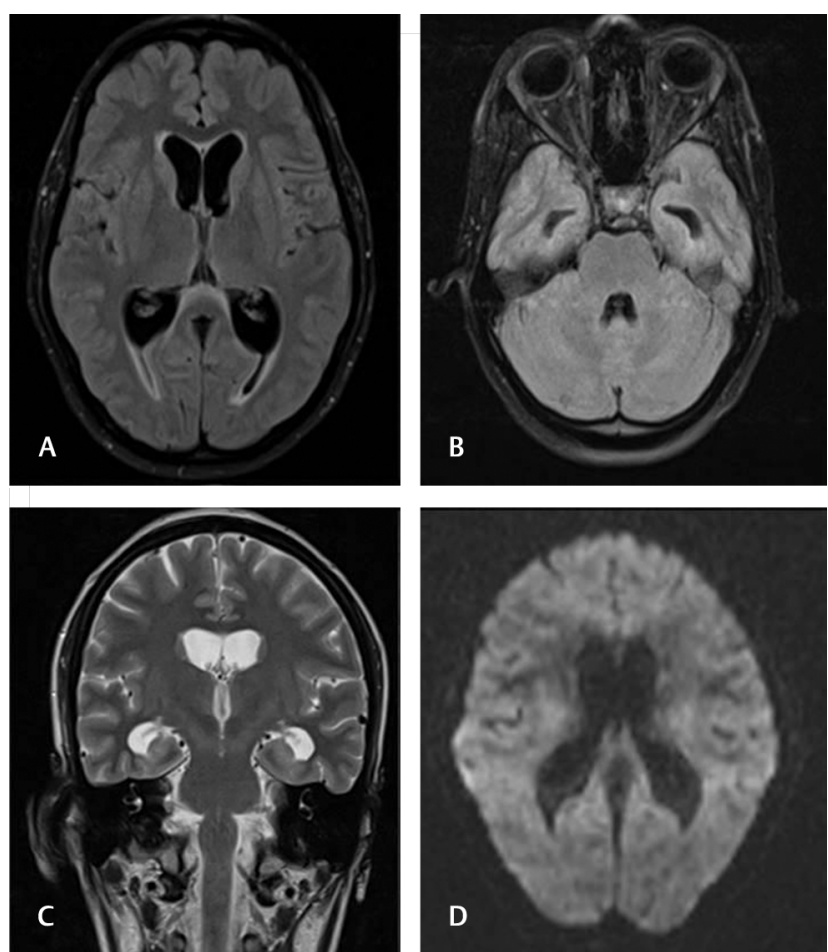

Fig. 8 Meningitis with hydrocephalus. A 18-year-old primigravida presented 1 week after delivery with headache and altered sensorium. Fluid-attenuated inversion recovery axial (A and $\mathbf{B}), \mathrm{T} 2$ coronal (C) sections showed moderate ventriculomegaly. Diffusion did not show any infarcts (D). Cerebrospinal fluid was suggestive of subacute meningitis and treated for tubercular meningitis. 
had positive for lupus anticoagulant test and other patient was negative for vasculitic workup.

\section{Infections/Tuberculosis}

During pregnancy, hormonal imbalance results in reduction in maternal immunity. The symptoms include headache, nausea, and focal neurological abnormalities. In susceptible population, tuberculosis may reactivate and flare up with central nervous system involvement. ${ }^{24-26}$ In fact, postpartum period is considered a form of immune restitution for reactivation tuberculosis. ${ }^{25}$ We had three patients: one had cerebritis with meningitis, second had granulomata, and third had only hydrocephalus with cerebrospinal fluid findings of subacute meningitis (-Figs. 7 and 8 ).

\section{Pituitary Diseases}

The maternal pituitary gland undergoes remarkable changes during pregnancy. Sheehan's syndrome results from deficiency of anterior pituitary hormone secondary to infarction and necrosis of the enlargement of pituitary physiologically in pregnancy. ${ }^{27,28}$ Enlargement of macroadenomas is common in women during pregnancy, especially who did not undergo surgery or radiation before conception. We had two cases of pituitary lesions-one had hemorrhage within physiological hyperplasia of the gland and another with preexisting macroadenoma developing hemorrhage and became symptomatic (-Figs. 9 and 10).
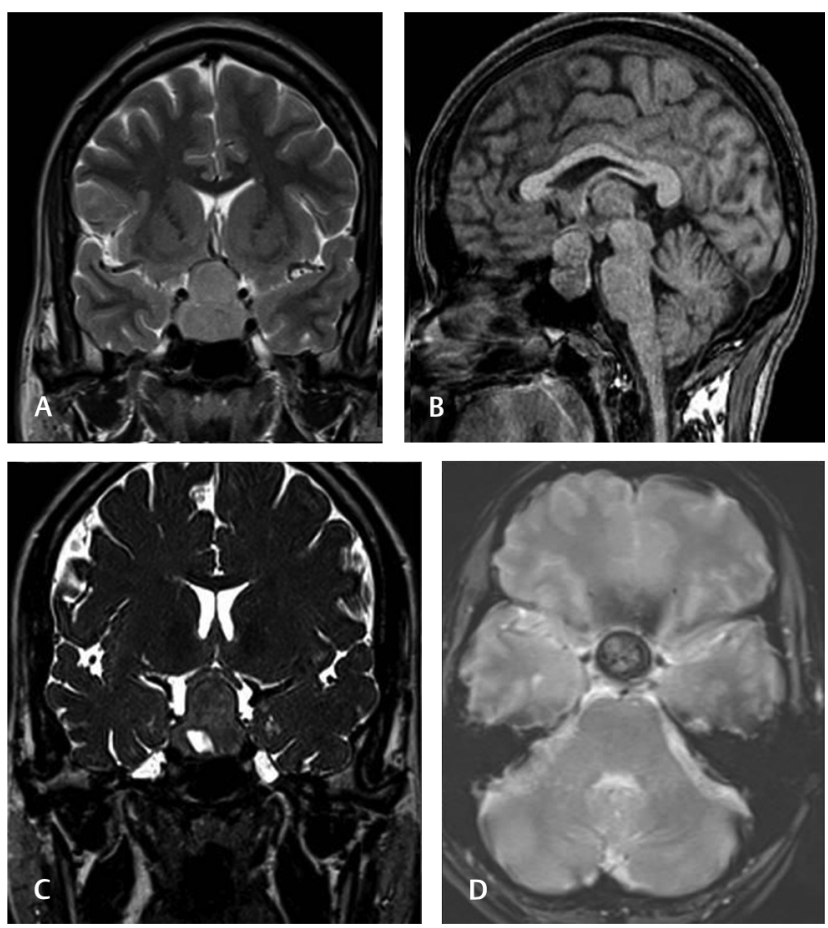

Fig. 9 Pituitary adenoma with hemorrhage. A 36-year-old G2P1 presented 10 days before labor with headache and altered sensorium. Magnetic resonance imaging $\mathrm{T} 2$ coronal and $\mathrm{T} 1$ sagittal sections showed sella-suprasellar mass probably pituitary macroadenoma. Heavily T2-weighted three-dimensional SPACE shows inferior cystic component and gradient-echo T2* hypointense hemorrhage.
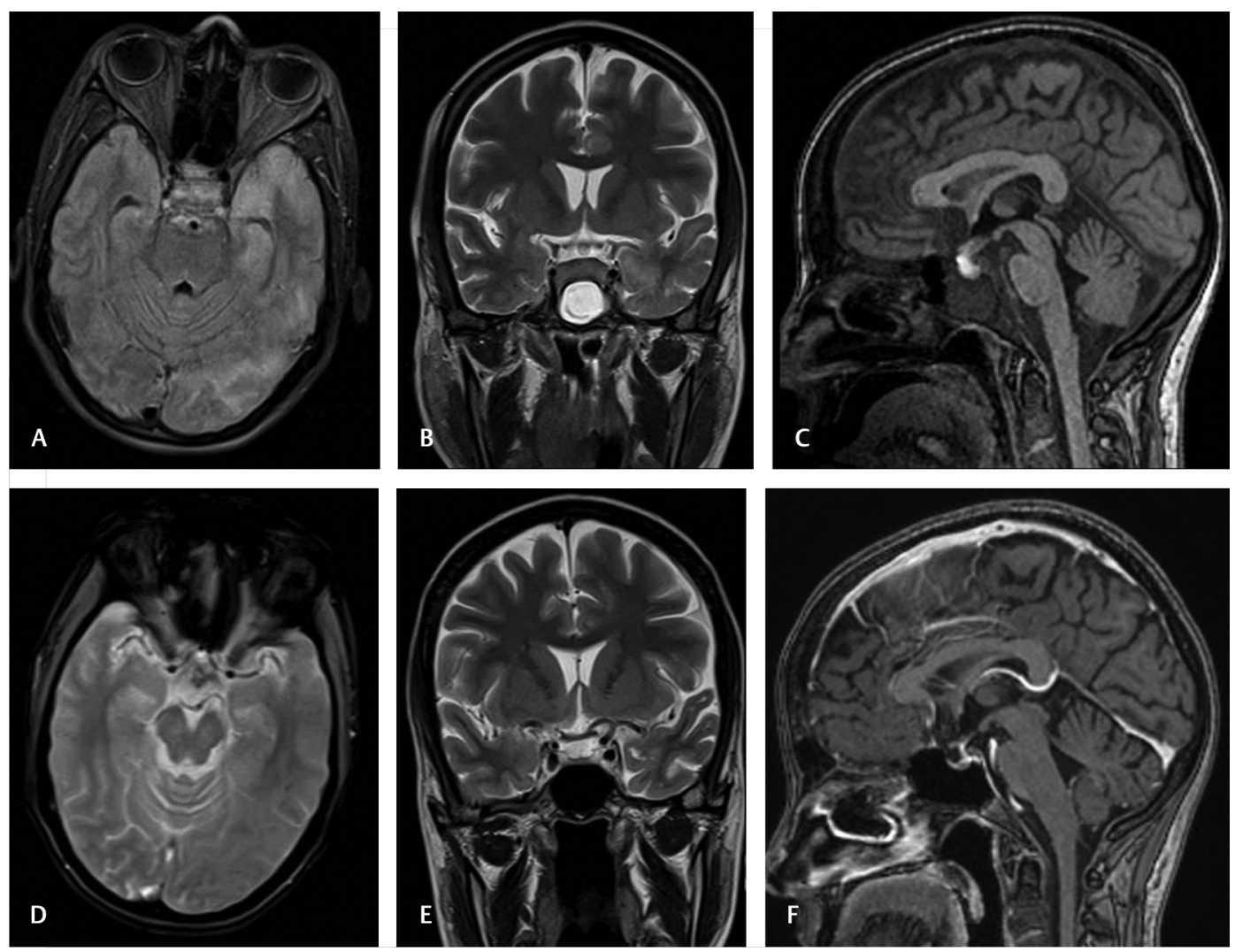

Fig. 10 Pituitary hemorrhage. A 25-year-old primigravida presented with headache and altered sensorium without any deficits. Fluid-attenuated inversion recovery axial and $\mathrm{T} 2$ coronal sections showed prominent pituitary with left temporal hyperintensities (A, B). The pituitary showed T1 hyperintensities $(\mathbf{C})$ and gradient-echo $\mathrm{T} 2{ }^{*}$ hypointensities $(\mathbf{D})$ suggestive of hemorrhage. Follow-up Magnetic resonance imaging (E and $\mathbf{F}$ ) after 3 months showed atrophy of anterior pituitary. 

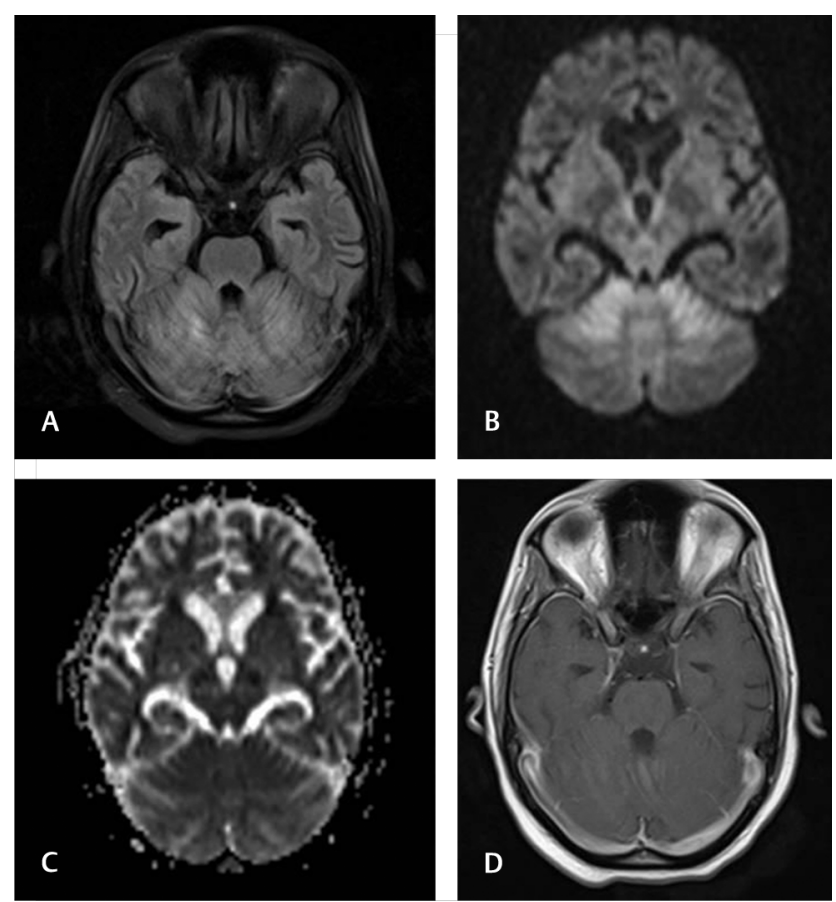

Fig. 11 Cerebellitis. A 19-year-old primigravida presented 4 days after labor with encephalopathy and ataxia. Fluid-attenuated inversion recovery axial (A), diffusion, (B) and apparent diffusion coefficient (C) images showing symmetric cerebellar hemispheric hyperintensities with diffusion restriction. No enhancement is noted (D).

\section{Cerebellitis}

Acute cerebellitis is uncommon in adults and can present as postinfectious or as part of viral infections like varicella, Ebstein-Barr virus, and others. ${ }^{29,30}$ MRI findings include cerebellar parenchymal T2-FLAIR hyperintensities with or without diffusion restriction. We had one patient who presented after 4 days with ataxia and MRI showed features of cerebellitis (-Fig. 11).

\section{Transient Splenial Lesion}

Transient splenial lesion in pregnancy is evolving and yet-to-be-defined entity. It has been ascribed to alteration in blood-brain barrier in conditions like hypertensive encephalopathy, preeclampsia, PRES, seizures, and drug toxicity. ${ }^{31,32}$ It has also been reported as part of PRES and postpartum angiopathy. However, it was an isolated finding in a 23-year-old primigravida who presented 3 days after labor (-Fig. 12).

\section{Postictal Edema}

Postictal edema may be present in imaging done immediately after seizure episode or with status epilepticus. ${ }^{33,34}$ In two patients of our series, isolated subcortical white matter edema was noted without any other findings ( - Fig. 13). They did not have other features of PRES or infection.
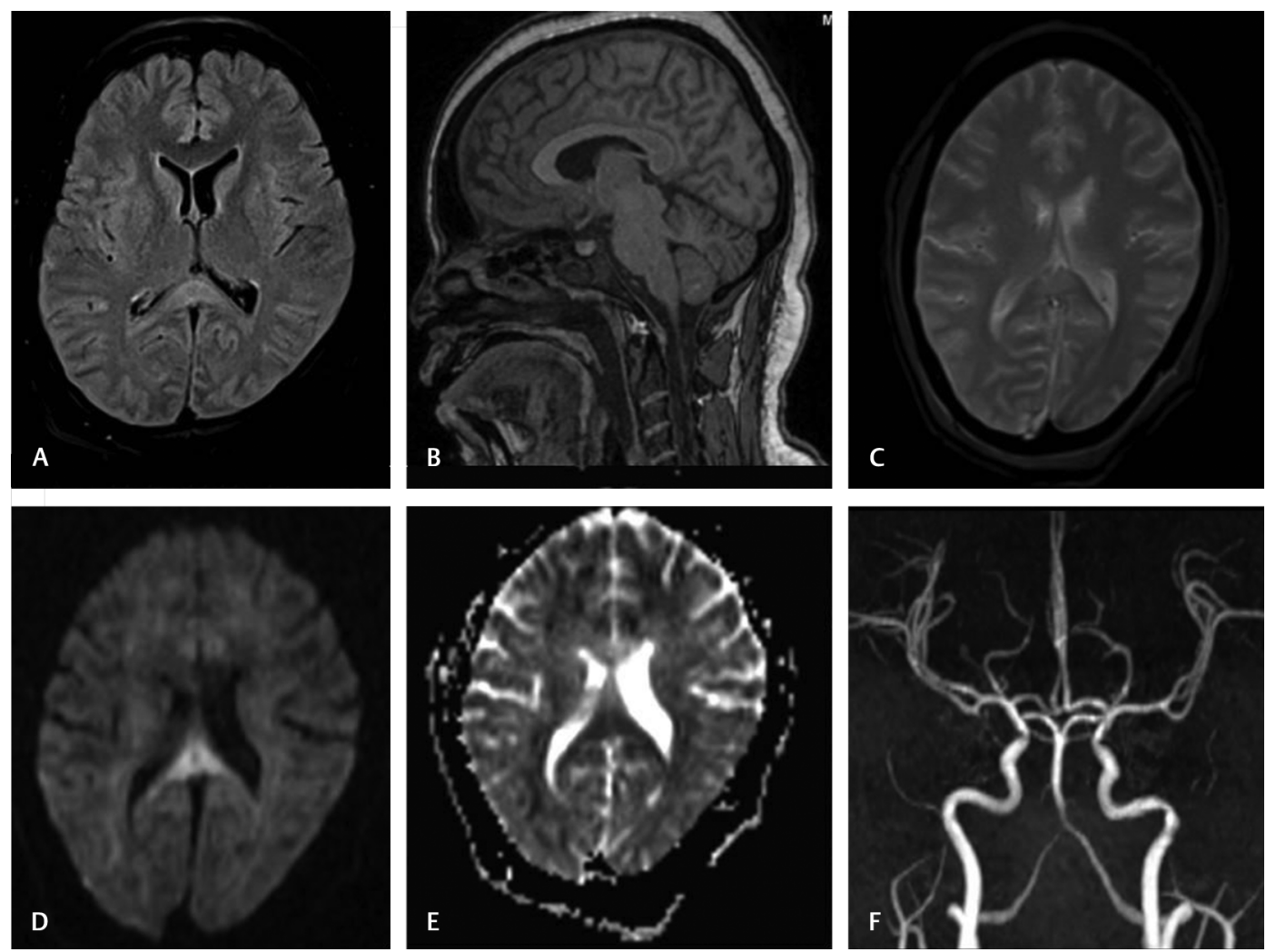

Fig. 12 Transient splenial lesion. A 23-year-old female presented 3 days after labor with encephalopathy. Fluid-attenuated inversion recovery axial (A) section shows hyperintense lesion in the splenium of corpus callosum. The lesion is hypointense in T1 sagittal (B), no hemorrhages in gradient-echo T2* image $(\mathbf{C})$ with diffusion restriction ( $\mathbf{D}$ and $\mathbf{E}$ ). Magnetic resonance imaging is normal $(\mathbf{F})$. Follow-up magnetic resonance imaging after a month showed resolution. 

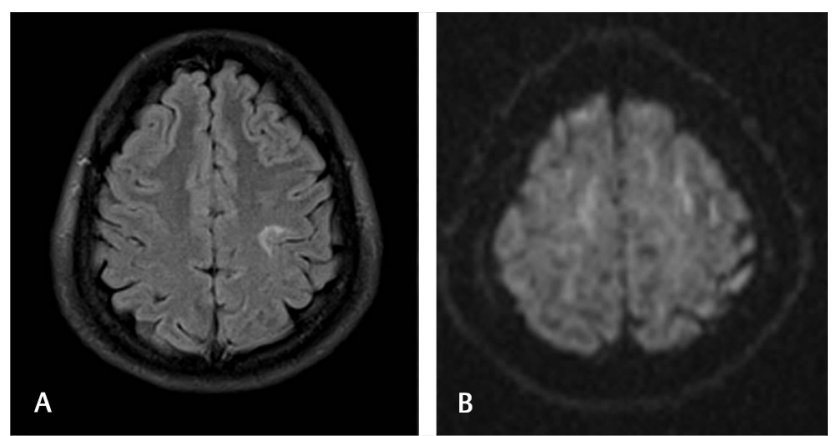

Fig. 13 Postictal edema. A 28-year-old primigravida presented 3 weeks after delivery with seizures and altered sensorium. Fluid-attenuated inversion recovery axial (A) section showing left perirolandic subcortical hyperintensities without diffusion restriction (B).

\section{Conflict of Interest}

None declared.

\section{References}

1 Lamy C, Sharshar T, Mas JL. [Cerebrovascular diseases in pregnancy and puerperium]. Rev Neurol (Paris) 1996; 152(6-7):422-440

2 Dineen R, Banks A, Lenthall R. Imaging of acute neurological conditions in pregnancy and the puerperium. Clin Radiol 2005;60(11):1156-1170

3 Hosley CM, McCullough LD. Acute neurological issues in pregnancyand theperipartum. Neurohospitalist2011;1(2):104-116

4 Al-Hayali RM, Al-Habbo DJ, Hammo MK. Peripartum neurological emergencies in a critical care unit. Neurosciences (Riyadh) 2008;13(2):155-160

5 Prabhu TR. Cerebrovascular complications in pregnancy and puerperium. J Obstet Gynaecol India 2013;63(2):108-111

6 Mortimer AM, Bradley MD, Likeman M, Stoodley NG, Renowden SA. Cranial neuroimaging in pregnancy and the post-partum period. Clin Radiol 2013;68(5):500-508

7 Junewar V, Verma R, Sankhwar PL, et al. Neuroimaging features and predictors of outcome in eclamptic encephalopathy: a prospective observational study. AJNR Am J Neuroradiol 2014;35(9):1728-1734

8 Al-Safi Z, Imudia AN, Filetti LC, Hobson DT, Bahado-Singh RO, Awonuga AO. Delayed postpartum preeclampsia and eclampsia: demographics, clinical course, and complications. Obstet Gynecol 2011;118(5):1102-1107

9 Lanska DJ, Kryscio RJ. Risk factors for peripartum and postpartum stroke and intracranial venous thrombosis. Stroke 2000;31(6):1274-1282

10 Gao H, Yang B-J, Jin L-P, Jia X-F. Predisposing factors, diagnosis, treatment and prognosis of cerebral venous thrombosis during pregnancy and postpartum: a case-control study. Chin Med J (Engl) 2011;124(24):4198-4204

11 Srinivasan K. Cerebral venous and arterial thrombosis in pregnancy and puerperium. A study of 135 patients. Angiology 1983;34(11):731-746

12 Geocadin RG, Razumovsky AY, Wityk RJ, Bhardwaj A, Ulatowski JA. Intracerebral hemorrhage and postpartum cerebral vasculopathy. J Neurol Sci 2002;205(1):29-34

13 Bittencourt LL, Maia AC Jr, da Rocha AJ. Postpartum angiopathy with reversible cerebral vasoconstriction syndrome (RCVS): imaging in diagnosis and follow-up. Arq Neuropsiquiatr 2011;69(2B) :412
14 Lemmens R, Smet S, Wilms G, Demaerel P, Thijs V. Postpartum RCVS and PRES with normal initial imaging findings. Acta Neurol Belg 2012;112(2):189-192

15 Fugate JE, Ameriso SF, Ortiz G, et al. Variable presentations of postpartum angiopathy. Stroke 2012;43(3):670-676

16 TsukimoriK,OchiH,YumotoY,etal. Reversible posteriorencephalopathy syndrome followed by MR angiography-documented cerebral vasospasm in preeclampsia-eclampsia: report of 2 cases. Cerebrovasc Dis 2008;25(4):377-380

17 Abbade JF, Peraçoli JC, Costa RAA, Calderon IdeM, Borges VTM, Rudge MVC. Partial HELLP syndrome: maternal and perinatal outcome. Sao Paulo Med J 2002;120(6):180-184

18 Padden MO. HELLP syndrome: recognition and perinatal management. Am Fam Physician 1999;60(3):829-836, 839

19 Tetsuka S, Nonaka H. Importance of correctly interpreting magnetic resonance imaging to diagnose posterior reversible encephalopathy syndrome associated with HELLP syndrome: a case report. BMC Med Imaging 2017;17(1):35

20 Pazhayattil GS, Rastegar A, Brewster UC. Approach to the diagnosis and treatment of hyponatremia in pregnancy. Am J Kidney Dis 2015;65(4):623-627

21 Saroja AO, Naik KR, Mali RV, Kunam SR. 'Wine Glass' sign in recurrent postpartum hypernatremic osmotic cerebral demyelination. Ann Indian Acad Neurol 2013;16(1):106-110

22 Ducloy-Bouthors AS, Trillot N. [Risk factors of thromboembolism associated with pregnancy and the puerperium. Role of inherited and acquired thrombophilia]. Ann Med Interne (Paris) 2003;154(5-6):295-300

23 Gómez-Puerta JA, Cervera R, Espinosa G, Bucciarelli S, Font J. Pregnancy and puerperium are high susceptibility periods for the development of catastrophic antiphospholipid syndrome. Autoimmun Rev 2006;6(2):85-88

24 Chan KH, Ho PL, Cheung RT, et al. Tuberculous meningitis with tuberculomata presenting as postpartum pyrexia of unknown origin. Hosp Med 2003;64(5):306-307

25 Cheng VC, Woo PC, Lau SK, et al. Peripartum tuberculosis as a form of immunorestitution disease. Eur J Clin Microbiol Infect Dis 2003;22(5):313-317

26 Brandstetter RD, Murray HW, Mellow E. Tuberculous meningitis in a puerperal woman. JAMA 1980;244(21):2440

27 Schrupp Berg HL, Edlow JA. Post-partum pituitary apoplexy: a case report. Intern Emerg Med 2007;2(4):311-314

28 Pivonello R, De Martino MC, Auriemma RS, et al. Pituitary tumors and pregnancy: the interplay between a pathologic condition and a physiologic status. J Endocrinol Invest 2014;37(2):99-112

29 Van Samkar A, Poulsen MNF, Bienfait HP, Van Leeuwen RB. Acute cerebellitis in adults: a case report and review of the literature. BMC Res Notes 2017;10(1):61010.1186/s13104-017-2935-8

30 De Bruecker Y, Claus F, Demaerel P, et al. MRI findings in acute cerebellitis. Eur Radiol 2004;14(8):1478-1483

31 Takahashi Y, Hashimoto N, Tokoroyama H, et al. Reversible splenial lesion in postpartum cerebral angiopathy: a case report. J Neuroimaging 2014;24(3):292-294

32 Yang Q Chang C-C, Liu M, Yu Y-Q. Sequential occurrence of eclampsia-associated posterior reversible encephalopathy syndrome and reversible splenial lesion syndrome (a case report): proposal of a novel pathogenesis for reversible splenial lesion syndrome. BMC Med Imaging 2019;19(1):3510.1186/s12880-019-0323-7

33 Kim NY, Baek HJ, Choi DS, et al. MR Findings of seizure-related cerebral cortical lesions during peri-ictal period. iMRI Investigative MRI 2017;21:82-90

34 Williams JA, Bede P, Doherty CP. An exploration of the spectrum of peri-ictal MRI change; a comprehensive literature review. Seizure 2017;50:19-32 